\title{
Breast cancer pharmacogenomics: where we
}

\section{are going}

\section{"As the science of genomics has matured, it is increasingly seen as a tool used in clinical practice to design new treatments and to tailor current treatments more effectively."}

\section{KEYWORDS: aromatase inhibitors " breast cancer " CYP2D6 " pharmacogenetics - tamoxifen}

There is perhaps no field of therapeutics in which personalized medicine has been more successfully implemented than in breast cancer. The routine use of estrogen receptor, progesterone receptor and HER-2 testing supports the value of biomarker-driven therapeutics in cancer in general. In addition, the use of somatic and transcriptomic arrays to guide therapy has been pioneered in the treatment of breast cancer. These advances have resulted in quantifiable improvements in the lives of patients with breast cancer, such that life expectancy of a Caucasian woman diagnosed early with low-grade disease in her mid 50s is 15 years or more.

It might reasonably be asked why we need even more progress, but the worldwide burden of this disease remains huge, such that many women diagnosed at a younger age or with more advanced disease, or who are of African or Asian ancestry have much worse prognoses with many dying within two years of presentation. Breast cancer worldwide remains the second most lethal cancer in women, responsible for more than 1 million premature deaths per year worldwide, and for healthcare costs that are astronomic by any measure, not least because the disease affects so many women in their prime. This is particularly true in Asia, where the incidence of breast cancer is rising rapidly, and the diagnosis of breast cancer is made approximately 10 years earlier than that in the western world, and where it is predominantly a disease of premenopausal women [1]. It follows that optimization of existing therapies is critical, and the development of new and tolerable therapies remains a high priority. For this reason, efforts to identify biomarkers that further focus treatment remain crucial and for this reason, this issue of pharmacogenomics is focused on the ongoing effort around the world to do so.
The broad science of pharmacogenomics and its associated technology is well placed to address the need for improved therapy, through the identification of new markers of disease response and through the ability of pharmacogenomics science to point the way to new mechanisms of therapy. With that said, there are many obstacles ahead, and while it is clear that genomic approaches will represent an important part of future clinical prediction, it is equally clear that genomic approaches are most powerful when linked to clinical and other predictors.

The challenges involved in the use of germline genetic biomarkers are illustrated by the story of tamoxifen pharmacogenomics. The possibility that germline genetic predictors of drug response might be a valuable means of identifying patients most appropriate for tamoxifen therapy was embraced by the scientific community worldwide. The initial observation in 2003, by the Consortium on Breast Cancer Pharmacogenomics [2,3], was that inherited variation in the activity of CYP2D6 alters serum concentrations of the most prominent antiestrogenic metabolite of tamoxifen, endoxifen, has been validated by a large number of worldwide studies and is beyond dispute [4-6]. Unfortunately, it is equally clear that this genetic variation alone does not predict breast cancer outcomes. This illustrates the difficulty of conducting genetic biomarker studies relevant to therapeutics in cancer. Companion studies of subsets of samples from the largest adjuvant tamoxifen trials (ATAC and BIG 1-98) reported no significant association between CYP2D 6 genotype and breast cancer outcomes [7]. In a sense, this is not surprising, since tamoxifen is a complex drug with many metabolites that have many actions, and our simplistic assumption that all of its activity might be ascribed

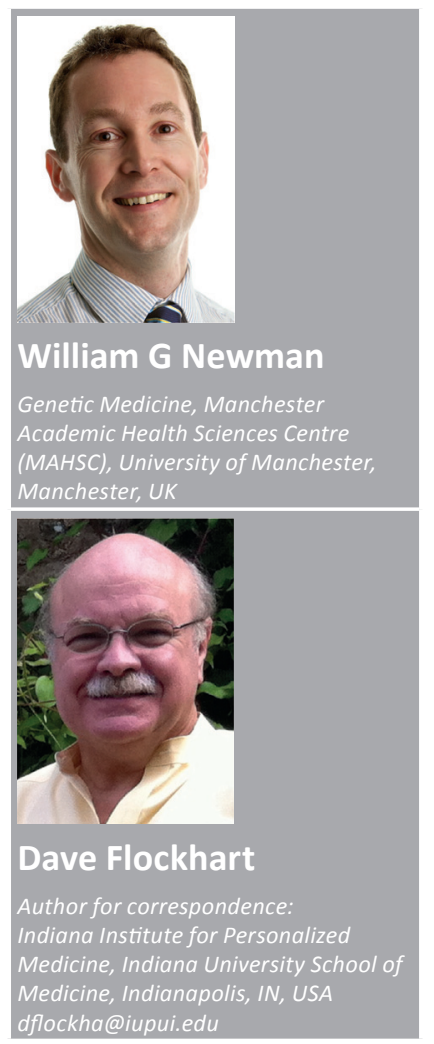

Future $\mathrm{fSg}$ 
to a single metabolite, with one antiestrogenic activity may well be flawed. The drug has been shown to act via PKC [8], and it has been recently shown that a number of prominent tamoxifen metabolites are active vasodilators [9] and also potent aromatase inhibitors [10]. The recent history of tamoxifen pharmacogenomics has emphasized a number of age-old lessons. Comparison of the results from different studies is compounded where different end points or differently defined end points are used. Robust, comprehensive genotyping methods to evaluate high-quality samples is critical, as is appropriate statistical and bioinformatic analysis. Great care should be taken in extrapolating somatic genomic data to the germline, and it is critical to power studies appropriately to allow the detection of clinically significant effects. Cancer pharmacogenomics is also complicated by the fact that different genetic signatures may be relevant in different settings: preventative, adjuvant and metastatic. Furthermore, we have already seen with irinotecan the importance of dose and regimen in the contribution of UGT1A1 genotype to toxicity [11].

It is perhaps in this prediction of treatment toxicity where the most notable short-term advances will be made. Genome-wide association studies have already identified variants relevant to the musculoskeletal side effects that occur with aromatase inhibitors [12].

\section{"The challenges involved in the use of germline genetic biomarkers are illustrated by the story of tamoxifen pharmacogenomics."}

As the science of genomics has matured, it is increasingly seen as a tool used in clinical practice to design new treatments and to tailor current treatments more effectively. This is evident in the interviews with Richard Weinshilboum and Alan Ashworth [13,14]. The articles by Kristen D
Hadfield and William Newman [15], Howard McLeod and Christine M Walko [16], and Anna González-Neira [17] address developing tests to allow more targeted treatment with aromatase inhibitors, tamoxifen and chemotherapy, respectively. The cutting edge of future approaches is illustrated by the editorial by Milan Radovic on the use of next-generation sequencing of both the germline genome and the somatic transcriptome to identify new drugable targets [18] Broader approaches beyond genetic profiling are required to personalize breast cancer treatment. In line with this, the article on the use of miRNA by Justin Stebbing points the way towards a new approach that has the potential to provide analytically robust biomarkers [19].

This issue also illustrates the ongoing role of pharmacogenomics in mechanisms: Hiltrud Brauch's article on sex hormone metabolism pharmacogenomics [20] and the excellent article by Toshihisa Ishikawa on transporter pharmacogenomics illustrate the great value that this science has to offer to the broader biological questions involved [21].

As is the case with any biomarker, its real value in the clinic critically depends on its place relative to existing predictors, and so Alastair Thompson's article on the importance of nonpharmacogenetic factors and adherence to therapy is a fitting bookend that places the science of pharmacogenomics in clinical context [22].

Financial \& competing interests disclosure

$D$ Flockhart reports funding for investigator-driven research by Pfizer, Inc. and Novartis, Inc. He serves on the Scientific Advisory Boards of Medco, Inc., and of The Coriell Institute for Medical Research. The authors have no other relevant affiliations or financial involvement with any organization or entity with a financial interest in or financial conflict with the subject matter or materials discussed in the manuscript apart from those disclosed.

No writing assistance was utilized in the production of this manuscript.

\section{References}

1 Ma GX, Yin L, Gao W, Tan Y, Liu R, Fang C, Ma XS. Workplace-based breast cancer screening intervention in china. Cancer Epidemiol Biomarkers Prev. 21(2), 358-367 (2012).

2 Stearns V, Johnson MD, Rae JM et al. Active tamoxifen metabolite plasma concentrations after coadministration of tamoxifen and the selective serotonin reuptake inhibitor paroxetine. J. Natl Cancer Inst. 95(23), 1758-1764 (2003).
3 Jin Y, Desta Z, Stearns V et al. CYP2D6 genotype, antidepressant use, and tamoxifen metabolism during adjuvant breast cancer treatment. J. Natl Cancer Inst. 97(1), 30-39 (2005).

4 Kiyotani K, Mushiroda T, Nakamura Y, Zembutsu H. Pharmacogenomics of tamoxifen: roles of drug metabolizing enzymes and transporters. Drug Metab. Pharmacokinet. 27(1), 122-131 (2012).

5 Lim JS, Chen XA, Singh O et al. Impact of CYP2D6, CYP3A5, CYP2C9 and CYP2C19 polymorphism on tamoxifen pharmacokinetics in Asian breast cancer patients. Br. J. Clin. Pharmacol. 71(5), 737-750 (2011).

6 Mürdter TE, Schroth W, Bacchus-Gerybadze L et al. Activity levels of tamoxifen metabolites at the estrogen receptor and the impact of genetic polymorphisms of phase I and II enzymes on their concentration levels in plasma. Clin. Pharmacol. Ther. 89(5), 708-717 (2011).

7 Rae JM. Personalized tamoxifen: what is the best way forward? J. Clin. Oncol. 29(24), 3206-3208 (2011).

8 Kuo JR, Wang CC, Huang SK, Wang SJ. Tamoxifen depresses glutamate release 
through inhibition of voltage-dependent $\mathrm{Ca} 2+$ entry and protein kinase $\mathrm{C} \alpha$ in rat cerebral cortex nerve terminals. Neurochem. Int. 60(2), 105-114 (2012).

9 Montenegro MF, Ceron CS, Salgado MC, Desta Z, Flockhart DA, Tanus-Santos JE. Tamoxifen and its metabolites cause acute vasorelaxation of aortic rings by inducing vasodilator prostanoid synthesis. J. Cardiovasc. Pharmacol. 58(6), 647-653 (2011).

10 Lu WJ, Desta Z, Flockhart DA. Tamoxifen metabolites as active inhibitors of aromatase in the treatment of breast cancer. Breast Cancer Res. Treat. 131(2), 473-481 (2012).

11 Hoskins JM, Goldberg RM, Qu P, Ibrahim JG, McLeod HL.UGT1A1*28 genotype and irinotecan-induced neutropenia: dose matters. J. Natl Cancer Inst. 99(17), 1290-1295 (2007).

12 Ingle JN, Schaid DJ, Goss PE et al. Genomewide associations and functional genomic studies of musculoskeletal adverse events in women receiving aromatase inhibitors. J. Clin. Oncol. 28(31), 4674-4682 (2010).

13 Weinshilboum RM. An interview with Pharmacogenomics for the breast cancer special focus issue. Pharmacogenomics 13(6), 655-658 (2012).

14 Ashworth A. Interview with Pharmacogenomics for the breast cancer special focus issue. Pharmacogenomics 13(6), 651-654 (2012).

15 Hadfield KD, Newman WG. Pharmacogenetics of aromatase inhibitors. Pharmacogenomics 13(6), 699-707 (2012).

16 Walko CM, McLeod H. Use of CYP2D6 genotyping in practice: tamoxifen dose adjustment. Pharmacogenomics 13(6), 691-697 (2012).

17 González-Neira A. Pharmacogenetics of chemotherapy efficacy in breast cancer. Pharmacogenomics 13(6), 677-690 (2012).
18 Radovich M. Next-generation sequencing in breast cancer: translational science and clinical integration. Pharmacogenomics 13(6), 637-639 (2012).

19 Krell J, Frampton AE, Jacob J, Castellano L, Stebbing J. miRNAs in breast cancer: ready for real time? Pharmacogenomics 13(6), 709-719 (2012).

20 Justenhoven C, Obazee O, Brauch $\mathrm{H}$. The pharmacogenomics of sex hormone metabolism: breast cancer risk in menopausal hormone therapy. Pharmacogenomics 13(6), 659-675 (2012).

21 Ishikawa T. Recent advances in pharmacogenomics of $\mathrm{ABC}$ transporters involved in breast cancer therapy. Pharmacogenomics 13(6), 633-636 (2012).

22 McCowan C, Thompson AM. The importance of non-pharmacogenetic factors in endocrine therapy. Pharmacogenomics 13(6), 721-728 (2012). 